\title{
Impact of collection, isolation and storage methodology of circulating microvesicles on flow cytometric analysis
}

\author{
FANCONG KONG $^{1 *}$, LIMING ZHANG $^{2 *}$, HONGXIANG WANG ${ }^{3}$, GUOLIN YUAN $^{4}$, \\ ANYUAN GUO $^{5}$, QIUBAI LI ${ }^{1}$ and ZHICHAO CHEN ${ }^{1}$ \\ ${ }^{1}$ Institute of Hematology, Union Hospital, Tongji Medical College, Huazhong University of Science and Technology, \\ Wuhan, Hubei 430022; ${ }^{2}$ Department of Hematology, Jingzhou Central Hospital, Jingzhou, Hubei 434020; \\ ${ }^{3}$ Department of Hematology, Wuhan Central Hospital, Wuhan, Hubei 430012; ${ }^{4}$ Department of Hematology, \\ Xiangyang Central Hospital, The Affiliated Hospital of Hubei University of Arts and Science, Xiangyang, \\ Hubei 441021; ${ }^{5}$ Department of Biomedical Engineering, College of Life Science and Technology, \\ Huazhong University of Science and Technology, Wuhan, Hubei 430074, P.R. China
}

Received December 22, 2014; Accepted July 28, 2015

DOI: $10.3892 /$ etm.2015.2780

\begin{abstract}
Microvesicles (MVs) in body fluids participate in a variety of physical and pathological processes, and are regarded as potential biomarkers for numerous diseases. Flow cytometry (FCM) is among the most frequently used techniques for MV detection. However, different handling methods unavoidably cause pre-analytical variations in the counts and sizes of MVs determined by FCM. The aim of the present study was to investigate the effect of centrifugation, storage conditions and anticoagulant on MV measurements. Blood samples were obtained from 13 healthy donors, including 4 women and 9 men. Calcein-AM staining was used to label MVs and assess the impact of pre-analytical preparation, including centrifugation, and storage conditions on MV measurements obtained using FCM. The range of factors investigated for comparison included: Platelet-free plasma (PFP) stored at $-80^{\circ} \mathrm{C}$ for 1 or 4 weeks; MVs stored at $4^{\circ} \mathrm{C}$ for 3-4 days or 1 week; MVs frozen at $-80^{\circ} \mathrm{C}$ for 1 or 4 weeks; and anticoagulants, either heparin or ethylenediaminetetraacetic acid (EDTA). No statistically
\end{abstract}

Correspondence to: Dr Zhichao Chen or Dr Qiubai Li, Institute of Hematology, Union Hospital, Tongji Medical College, Huazhong University of Science and Technology, 1,277 Jiefang Road, Wuhan, Hubei 430022, P.R. China

E-mail: chenzhichao@hust.edu.cn

E-mail: doctorlee007@sina.com

*Contributed equally

Abbreviations: MVs, microvesicles; FCM, flow cytometry; EDTA, ethylenediaminetetraacetic acid; PBS, phosphate-buffered saline; PFP, platelet-free plasma; PS, phosphatidylserine; AFM, atomic force microscopy; DLS, dynamic light scattering; TEM, transmission electron microscopy

Key words: microvesicles, calcein-AM, flow cytometry, pre-analytical preparation significant differences in MV counts were detected between the two centrifugation speeds $(16,000$ and $20,500 \mathrm{x}$ g) or among the three centrifugation times $(15,30$ and $60 \mathrm{~min})$ investigated. Similarly, no significant differences were noted in MV counts between the two anticoagulants tested (heparin and EDTA). However, the storage of PFP or MVs in heparin-anticoagulated plasma for different periods markedly affected the detected MV counts and size distribution. The counts and sizes of MVs from EDTA-anticoagulated plasma were only affected when the $\mathrm{MVs}$ were frozen at $-80^{\circ} \mathrm{C}$ for 4 weeks. In conclusion, calcein-AM is able to efficiently identify MVs from plasma and may be an alternative to Annexin V for MV staining. EDTA preserves the MV counts and size more accurately compared with heparin under calcein-AM staining. PFP centrifuged at $16,000 \mathrm{x} \mathrm{g}$ for $15 \mathrm{~min}$ is sufficient to isolate MVs, which enables the batch processing of samples. PFP, rather than MVs alone, appears to be the preferable mode of sample storage, as MVs stored in PFP were less affected by storage temperature and duration. The present study provides a methodology for MV collection, storage and isolation, to facilitate further investigation of MVs as biomarkers in disease.

\section{Introduction}

Microvesicles (MVs) are a heterogeneous group of membrane vesicles, with a size range of 100-1,000 nm, which are shed by various cell types in physiological and pathological conditions (1). MVs have been identified in a number of body fluids, including the plasma, serum, urine, saliva, breast milk, amniotic fluid and ascites (2-7). The counts and contents of MVs in circulation depend on the cells of origin and the stimuli that trigger MV production. Studies observing plasma by flow cytometry (FCM) have revealed correlations between MV counts and other characteristics of human hematologic disorders (8), cardiovascular (9) and autoimmune diseases $(10)$, and cancer $(11,12)$. Therefore, circulating MVs represent promising biomarkers and may have diagnostic and prognostic value for diseases. 
However, due to different methods used for collection, storage and isolation, pre-analytical variations in MV detection are unavoidable (13-16). A number of anticoagulants are used in blood samples collected for MV analysis, including heparin $(13,17)$, acid-citrate-dextrose (18), ethylenediaminetetraacetic acid (EDTA) (14) and sodium citrate (19). Previous studies have reported that heparin and EDTA lead to conflicting results of MV count $(15,16)$. In addition, different centrifugation speeds and times are used to isolate MVs; however, the effect of different centrifugation conditions on MV count are unclear $(8,20)$. Furthermore, storage status, time and temperature may markedly affect MV counts, as observed in previous studies $(13,15)$. Recent studies have demonstrated that MV size distribution may differ in response to various forms of stress in vitro $(20,21)$, indicating the significance of MV size in diseases. However, the effects of sample collection procedures and processing on MV size distribution have not been well-characterized.

The aim of the present study was to investigate the impact of pre-analytical sample preparation procedures and conditions on the counts and size distribution of MVs assessed using FCM.

\section{Materials and methods}

Reagents. Calcein-AM (C1430) was obtained from Life Technologies (Carlsbad, CA, USA) and used for MV staining as previously described (20). Calibration beads of $1 \mu \mathrm{m}$ (L1030) and $3 \mu \mathrm{m}$ (LB30) were purchased from Sigma-Aldrich (St. Louis, MO, USA) to define the MV gate and calculate MV counts. A submicron bead calibration kit (no. 832) was obtained from Bangs Laboratories, Inc. (Fishers, IN, USA) to verify the resolution capabilities of the flow cytometer and define the size distribution of MVs. Phosphate-buffered saline (PBS) solution (SH30256.01B) was purchased from HyClone Corporation (Logan, UT, USA), and filtered through a $0.22-\mu \mathrm{m}$ filter (EMD Millipore, Billerica, MA, USA) to minimize interference from particles in PBS.

Blood sample collection. Optimization of MV detection was performed using samples from healthy individuals. Written informed consent was obtained from 13 donors (female, 4; male, 9; age range, 22-33 years; median age, 26 years) under a protocol approved by the local Institutional Review Board of Tongji Medical College (Wuhan, China). All participants fasted for $10 \mathrm{~h}$ prior to sample collection. A total of 26 blood samples were collected from the 13 donors and 21-gauge needles were used to place the samples in BD vacutainers (BD Biosciences, Franklin Lakes, NJ, USA) of which the inner wall were sprayed with heparin or EDTA. There were 2 samples from each donor and $2 \mathrm{ml}$ per sample. These samples were used for the evaluation of the effect of storage conditions and anticoagulant on MV counts and size distribution. To assess the impact of centrifugation speed and time, 8 specimens were collected from healthy individuals (female, 4; male, 4; age range, 24-26 years; median age, 25 years) and placed in BD vacutainer tubes with heparin sprayed on the inner wall, $2 \mathrm{ml}$ per sample. For all samples, the first $3 \mathrm{ml}$ of blood collected following venepuncture was discarded. All samples were centrifuged within $2 \mathrm{~h}$ from collection.
Preparation of platelet-free plasma (PFP). PFP was obtained from anticoagulated blood as previously described (15) with certain modifications. Briefly, whole blood samples were centrifuged at $2,500 \times \mathrm{g}$ for $30 \mathrm{~min}$ at $20^{\circ} \mathrm{C}$, and plasma was collected and centrifuged for an additional $30 \mathrm{~min}$ at $2,500 \mathrm{x} \mathrm{g}$. Next, the supernatant was collected and aliquots of $100 \mu \mathrm{lPFP}$ were stored at $-80^{\circ} \mathrm{C}$ for 1 or 4 week until use, or MVs were isolated immediately.

Isolation of MVs. MVs were isolated from PFP, that was stored at $-80^{\circ} \mathrm{C}$ and thawed rapidly to $37^{\circ} \mathrm{C}$ prior to use (13). The PFP was centrifuged at $20,500 \times \mathrm{g}$ for $60 \mathrm{~min}$ at $4^{\circ} \mathrm{C}$ to obtain a MV pellet, as described by Ghosh et al (8). The centrifuge tube was tipped to discard the supernatant, leaving the pellet at the bottom undisturbed. The MV pellet was resuspended in $100 \mu \mathrm{l}$ PBS by gentle vortexing for $20 \mathrm{sec}$ for immediate analysis using FCM, or storage at $4^{\circ} \mathrm{C}$ for 3-4 days or 1 week or or $-80^{\circ} \mathrm{C}$ for 1 or 4 week prior to analysis. Unless otherwise indicated, MVs freshly obtained from PFP without any storage were used as controls.

In order to investigate the effects of centrifugation speed and duration on MV analysis, PFP from heparin-anticoagulated blood was immediately centrifuged at $4{ }^{\circ} \mathrm{C}$ and 16,000 or $20,500 \mathrm{x} \mathrm{g}$, for 15,30 or $60 \mathrm{~min}$ to isolate MVs. MVs obtained at $20,500 \mathrm{x}$ g were regarded as the control in this section. The workflow is outlined in Fig. 1.

FCM analysis of MVs. Data were acquired and analyzed using a BD LSR II flow cytometer (BD Biosciences) equipped with FACSDiva software. Forward scatter (FSC) and side scatter (SSC) of light were set in a logarithmic scale, and the fluorescence channels were set at logarithmic gain. Calibration beads were used to set the MV gate and to calculate the MV counts.

In order to distinguish true events from electronic noise and increase the specificity of MV detection, events in the MV gate were further discriminated by labeling with calcein-AM (20). MVs were defined as particles $<1.0 \mu \mathrm{m}$ in diameter that exhibited positive staining for calcein-AM. Individual MV samples were labeled with $0.5 \mu \mathrm{l}$ calcein-AM (5 $\mu \mathrm{mol} / \mathrm{l})$ for $25-30 \mathrm{~min}$ in the dark and diluted to a final volume of $300 \mu 1$. The time and concentration employed was optimized by titration. MV samples prepared in PBS without calcein-AM were used as negative controls.

For calculation of MV counts, 3- $\mu$ m calibration beads $(0.5 \mu \mathrm{l})$ were added immediately prior to analysis. Gain settings were adjusted to place the beads in the top right corner for scatter. The equation $M V=G_{M V} \times T C /\left(G_{T C} \times V\right)$ was used to calculate the absolute counts of MVs in single staining, where $\mathrm{G}_{\mathrm{MV}}$ is the number of events in the MV gate, $\mathrm{G}_{\mathrm{TC}}$ is the number of events in the $3-\mu \mathrm{m}$ calibration bead gate and $\mathrm{TC}$ is the number of beads added to the sample of volume $\mathrm{V}(15,22,23)$. Data acquisition stopped when the number of events in $G_{T C}$ reached 100,000. The original data analysis was performed using FACSDiva software (version 6.1.2; BD Biosciences), and FlowJo software (version 7.6.2; Treestar, Inc., Palo Alto, CA, USA) was applied to analyze the size distribution.

Statistical analysis. Statistically significant differences were compared among groups using the independent-samples $t$-test. Analysis was performed using SPSS statistical software 

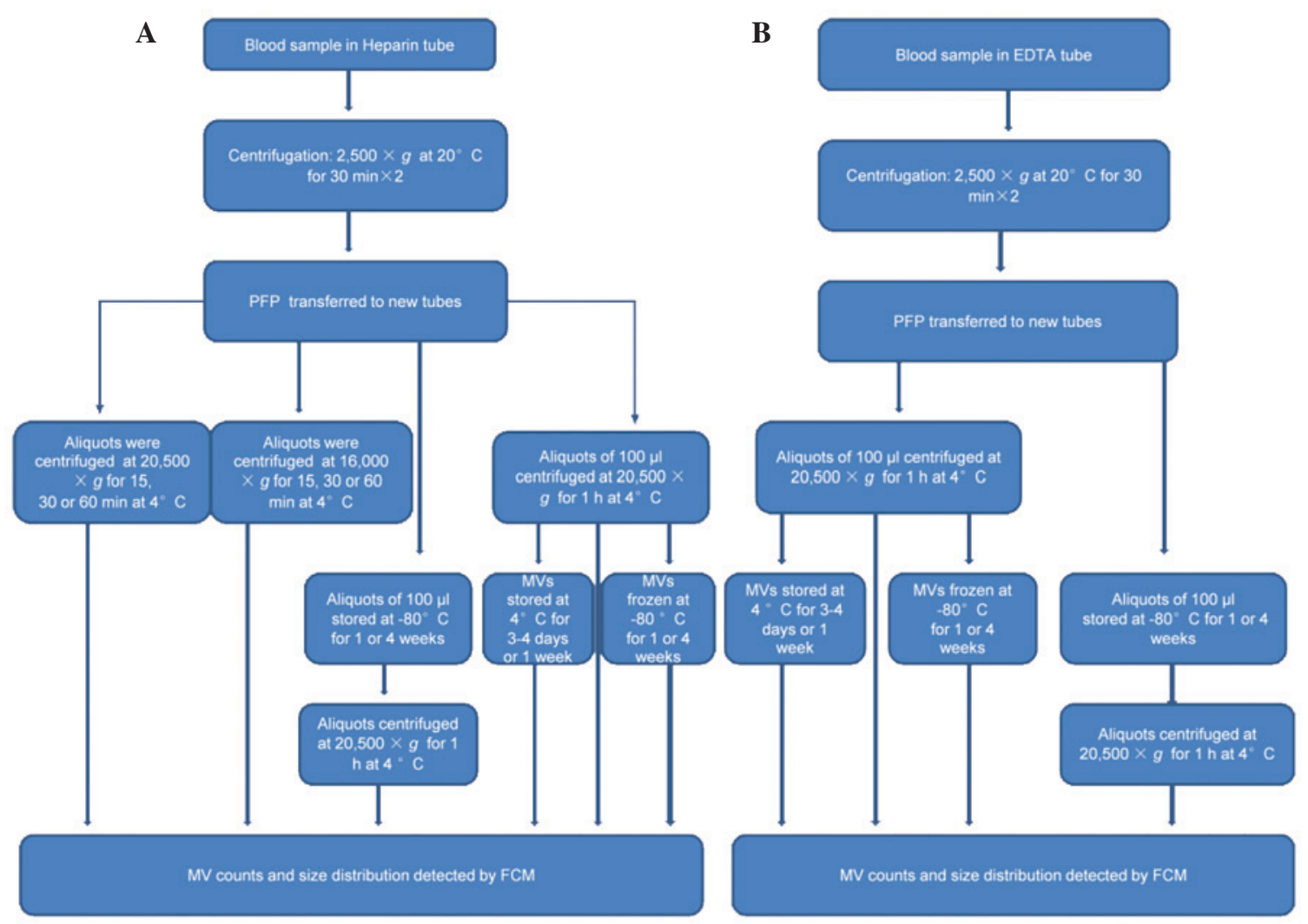

Figure 1. Sample treatment flowchart showing the experimental strategy for evaluating variables of collection, storage and isolation for MV counts and size distribution using FCM detection. (A) Blood samples were collected in BD Vacutainers tubes with heparin sprayed on the inner wall and centrifuged twice at 2,500 x g for $30 \mathrm{~min}$ to obtain PFP. A number of aliquots were centrifuged at 16,000 or $20,500 \mathrm{xg}$ for 15,30 or $60 \mathrm{~min}$ at $4^{\circ} \mathrm{C}$ to obtain $\mathrm{MVs}$, which were analyzed immediately to evaluate the influences of centrifugation speed and time on MV count and size distribution. Other PFP aliquots were centrifuged at $20,500 \mathrm{xg}$ for $1 \mathrm{~h}$ at $4^{\circ} \mathrm{C}$ to obtain MVs, or stored at $-80^{\circ} \mathrm{C}$ for various periods prior to isolation of the MVs. Subsequently, these MVs were analyzed immediately or stored at 4 or $-80^{\circ} \mathrm{C}$ for various periods until required for analysis using FCM. (B) Blood samples were collected in BD Vacutainers tubes with EDTA sprayed on the inner wall. Following double centrifugation at 2,500 x g for $30 \mathrm{~min}$ the supernatants were transferred to other tubes. Aliquots of $100 \mu 1$ were centrifuged at $20,500 \mathrm{xg}$ for $1 \mathrm{~h}$ at $4^{\circ} \mathrm{C}$ to obtain MVs, or stored at $-80^{\circ} \mathrm{C}$ for various periods prior to isolation of the MVs. MVs were analyzed immediately, or stored at 4 or $-80^{\circ} \mathrm{C}$ for various periods prior to analysis by FCM. EDTA, ethylenediaminetetraacetic acid; PFP, platelet-free plasma; MV, microvesicle; FCM, flow cytometry.

(version 16.0; SPSS, Inc., Chicago, IL, USA). Two-sided $\mathrm{P}$-values were used throughout. $\mathrm{P}<0.05$ was considered to indicate a statistically significant difference.

\section{Results}

Data acquisition, gating and counting strategy. Scatter events from size calibration beads of $0.2,0.5,0.8,1$ and $3 \mu \mathrm{m}$ were resolved from instrument noise using the BD LSR II flow cytometer (Fig. 2). Inspection of the scatter plot indicates that $0.2 \mu \mathrm{m}$ is the lower limit for bead detection (Fig. 2B). Calibration beads of $0.2,0.5$ and $0.8 \mu \mathrm{m}$ were additionally used to define the size distribution of MVs. As shown in Fig. 2D, MVs may be divided into three size ranges of 0.2-0.5, $0.5-0.8$ and $0.8-1.0 \mu \mathrm{m}$ in diameter. Fig. $2 \mathrm{E}$ is the histogram of the data presented in Fig. 2A, verifying the applicability of size distribution defined by the small beads.

In the present study, MVs were defined as particles that were $<1.0 \mu \mathrm{m}$ in diameter and positive for calcein-AM staining. As shown in Fig. 2F, events in the P6 gate were regarded as MVs from plasma. MV counts were calculated by reference to added $3-\mu \mathrm{m}$ calibration beads. According to the product information from Sigma-Aldrich, the number of particles per microliter of these beads is $6.8 \times 10^{6}$, and $0.5 \mu 1$ beads were added per experimental sample. Thus, the number of particles added per sample was $3.4 \times 10^{6}$. Data acquisition stopped when events in the P2 gate (3- $\mu \mathrm{m}$ calibration beads gate) reached 100,000; events in the P6 (MV) gate were recorded simultaneously. The number of MVs was calculated according to the formula described in the methods section.

$M V$ counts and size distribution are not affected by centrifugation speed or time. Various centrifugation speeds and times have been used in previous studies for MV isolation $(8,13,20)$; however, the effect of these variations in the parameters on MV counts and size distribution is unclear. In the present study, the differences arising from the various methods used for MV isolation were investigated. MVs isolated by centrifugation at 16,000 or $20,500 \mathrm{x} g$ for 15,30 or $60 \mathrm{~min}$ were analyzed using FCM. No statistically significant differences were detected in MV counts or size distribution between samples prepared at different centrifugation speeds, or among samples centrifuged for different times at identical speeds (Fig. 3).

Counts and size of MVs from heparin-anticoagulated samples vary with storage conditions. A number of studies have 

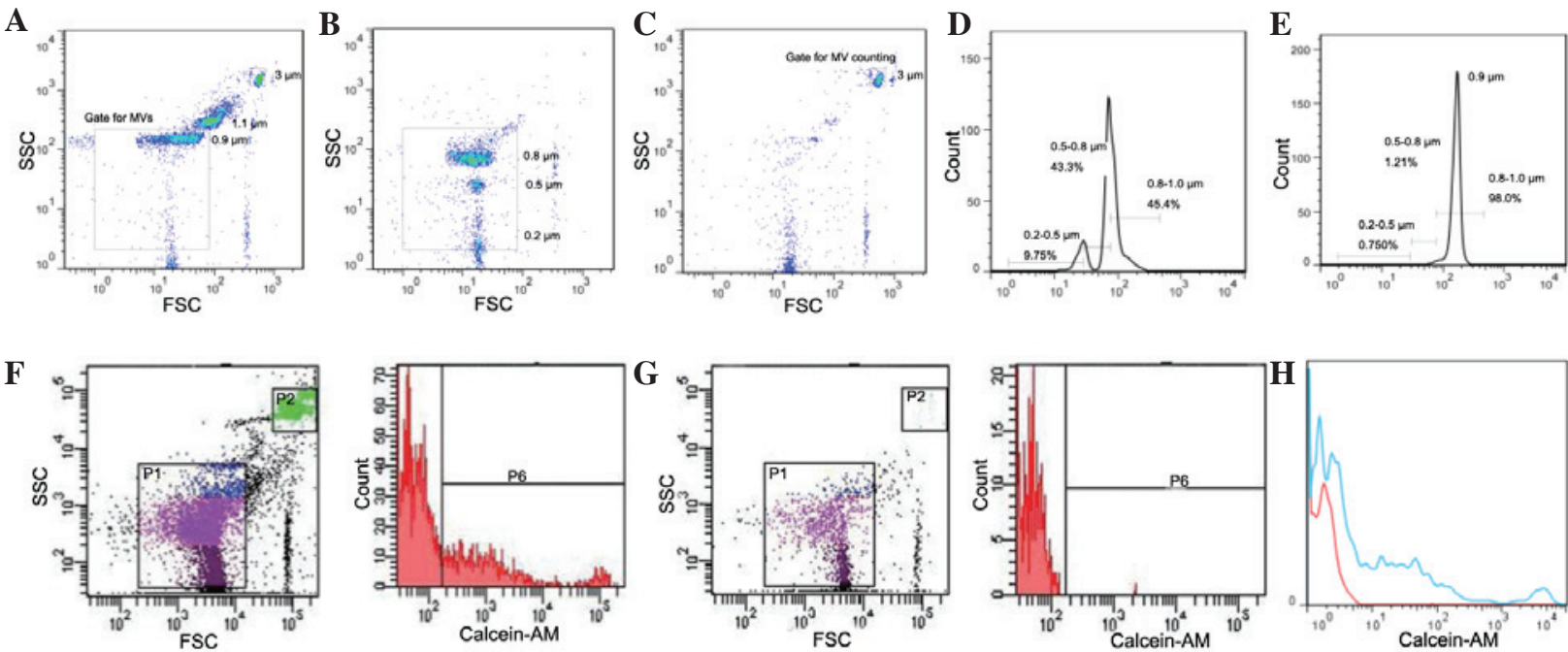

Figure 2. Gating and counting strategy. Representative dot plots and histograms illustrate gating and counting strategy for flow cytometric analysis of MVs. (A) $1 \mu \mathrm{m}$ (a mixture of 0.9 and $1.1 \mu \mathrm{m}$ ) beads from Sigma-Aldrich were used to set the MV gate and establish appropriate instrument settings. The MV gate was defined as $<1.0 \mu \mathrm{m}$. (B) A small bead calibration kit was used to verify the resolution capabilities of the flow cytometer, and to define the size distribution of MVs. (C) Representative dot plot for the 3- $\mu \mathrm{m}$ beads used for MV counting. (D) Histogram of size distributions from the data in Fig. 2B. (E) Histogram of size distribution from the data in Fig. 2A, verifying the applicability of Fig. 2D. (F) Representative sample of MV counting from the same individual as for the data in Fig. 2G. (G) An individual sample of MVs without calcein-AM staining was used as the calcein-AM negative control. (H) Histogram of the data in Fig. 2F, normalized against Fig. 2G. Red lines indicate data from Fig. 2G and blue lines indicate data from Fig. 2F. SSC, side scatter; FSC, forward scatter.
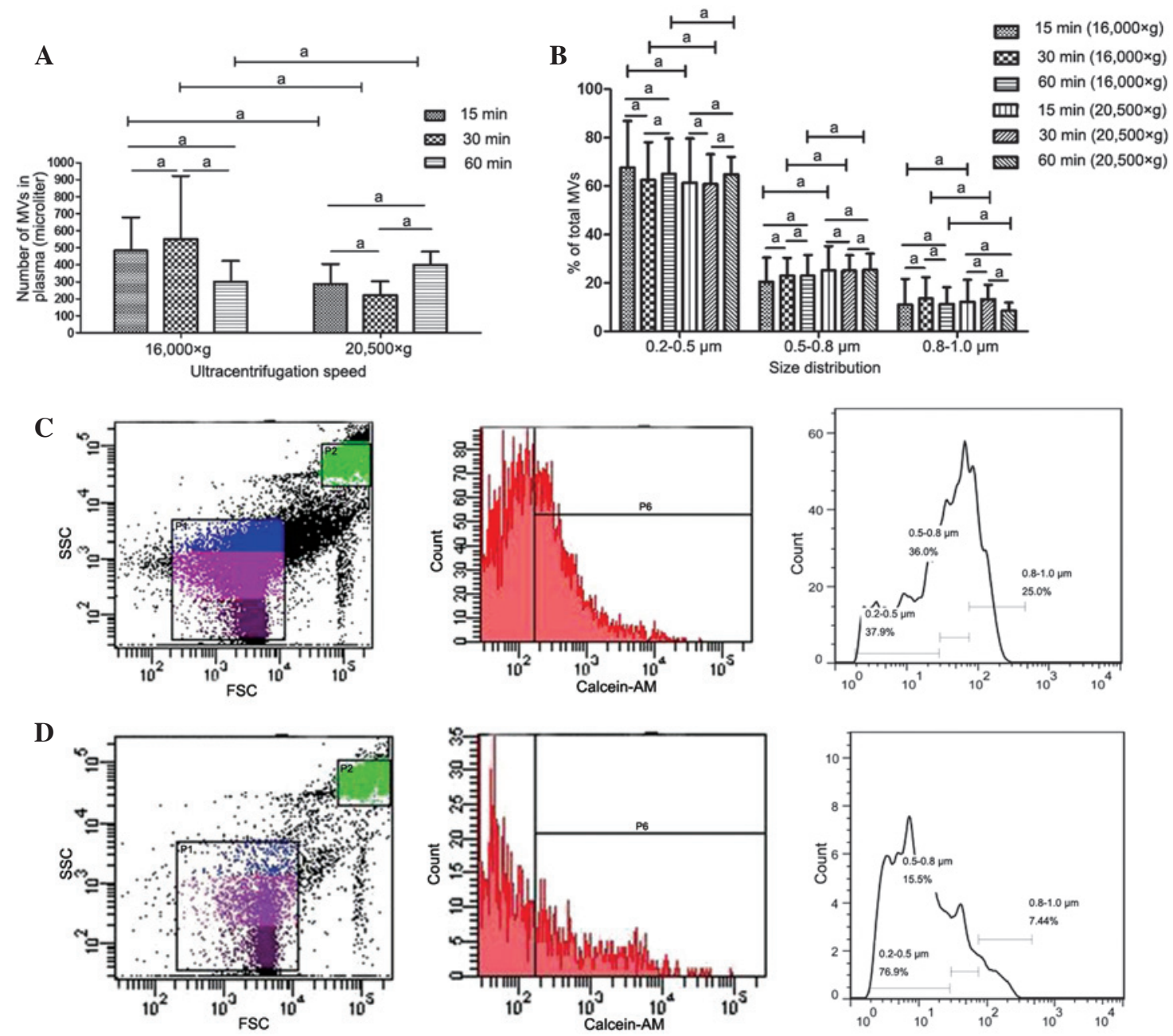

Figure 3. Impact of centrifugation speed and duration on MV counts and size distribution. Differences in (A) MV count and (B) size distribution, respectively. Also, representative dot plot and histogram showing the effects of centrifugation speed on MV count and size distribution for samples from a single individual are presented: (C) Samples centrifuged at 20,500 x g; and (D) samples centrifuged at 16,000 x g. Data are expressed as the mean \pm standard error of the mean. ${ }^{a}$ No statistically significant difference. MV, microvesicle; SSC, side scatter; FSC, forward scatter. 
A

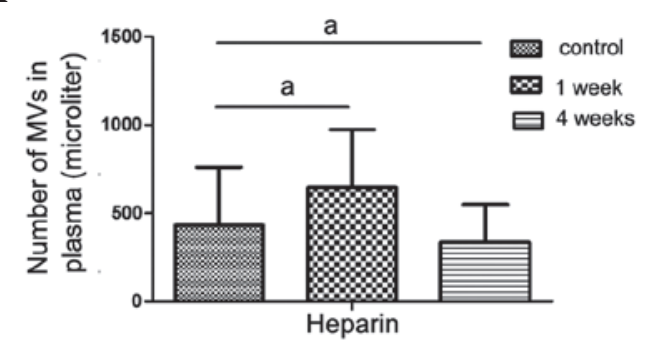

C
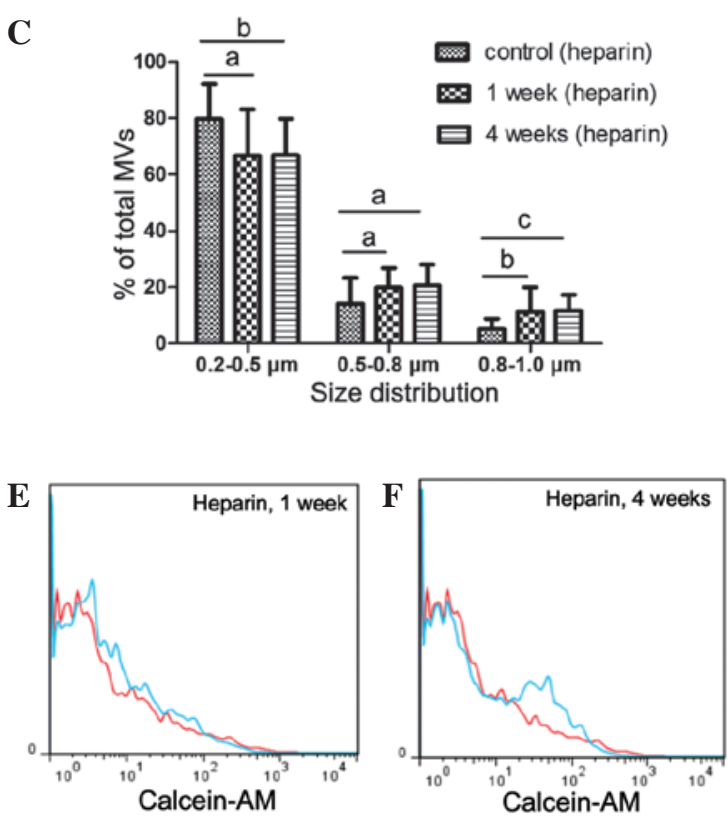

B

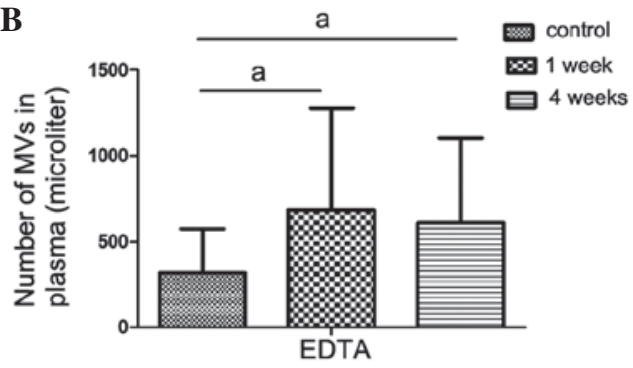

D

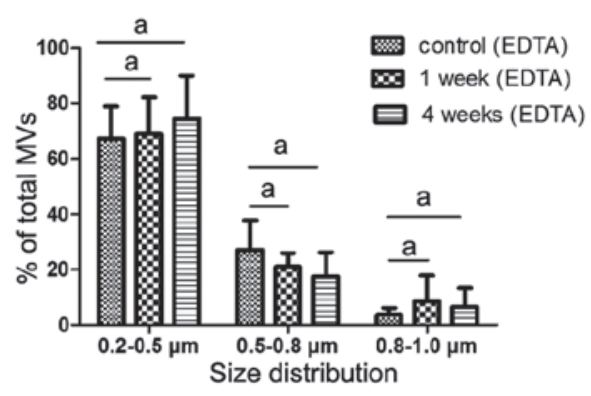

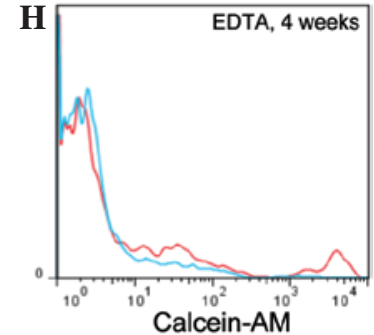

Figure 4. Impact of PFP storage at $-80^{\circ} \mathrm{C}$ for 1 or 4 weeks on MV counts and size distribution. Impact of (A) heparin and (B) EDTA on MV counts, as well as impact of (C) heparin and (D) EDTA on MV size distribution. Representative histograms of MV counts vs. control samples from a single individual are also shown (red lines, control; blue lines, experiment). Platelet-free plasma (PFP) from heparin-anticoagulated plasma at (E) 1 week and (F) 4 weeks. PFP from EDTA-anticoagulated plasma at $(\mathrm{G}) 1$ week and $(\mathrm{H}) 4$ weeks. Control samples were collected and centrifuged immediately to isolate MVs (i.e. there

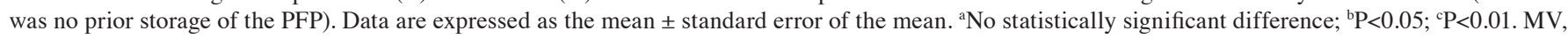
microvesicle; EDTA, ethylenediaminetetraacetic acid.

reported that heparin is able to preserve MV counts $(15,16)$ Therefore, in the present study, heparin was used as the primary anticoagulant in collected samples. In order to investigate the effect of storage conditions on MV counts and size distribution, the PFP or MVs were stored for different periods at various temperatures until analysis (Fig. 4A, C, E and F).

Counts and size of MVs from PFP stored at $-80^{\circ} \mathrm{C}$ for 1 or 4 weeks were compared with the control samples (MVs that were isolated immediately). Fig. 4C demonstrates that MV size, but not count, was significantly affected. After 1 week of storage of PFP, MV size was larger compared with that of the control samples, with an increased percentage of $0.8-1.0 \mu \mathrm{m}$ MVs observed compared with the control $(\mathrm{P}=0.025)$. After 4 weeks, the percentage of MVs distributed in the 0.8-1.0 $\mu \mathrm{m}$ group significantly increased $(\mathrm{P}=0.006)$, while the percentage in the $0.2-0.5 \mu \mathrm{m}$ group decreased compared with the control $(\mathrm{P}=0.038)$.

In addition to PFP, MVs is another mode of storage. It was observed that freezing MVs at $-80^{\circ} \mathrm{C}$ markedly affected the MV count and size (Fig. 5). MV counts significantly reduced after MVs were stored for 1 or 4 weeks at $-80^{\circ} \mathrm{C}$ ( 1 week, $\mathrm{P}=0.006$; 4 weeks, $\mathrm{P}<0.001)$ compared with the control, whereas the percentage of MVs in the $0.8-1.0 \mu \mathrm{m}$ bead size group increased
( 1 week, $\mathrm{P}=0.018 ; 4$ weeks, $\mathrm{P}=0.014$ ). By contrast, MV counts and size distribution did not change after MVs were stored at $4^{\circ} \mathrm{C}$ for 3-4 days or 1 week (Fig. 5A and B).

MVs from EDTA-anticoagulated samples. Although a number of studies $(15,16)$ have indicated that MVs from EDTA-anticoagulated plasma do not accurately evaluate the level of circulating MVs, EDTA is the most frequently used anticoagulant in clinical settings. On the basis of the protective effect of EDTA on blood cells, it was speculated that EDTA would protect MVs from storage influences and prolong safe storage time. Furthermore, the present study aimed to determine whether EDTA was able to function as a feasible alternative to heparin.

In the EDTA-anticoagulated PFP that was stored at $-80^{\circ} \mathrm{C}$ for 1 or 4 weeks, no statistically significant increase in MV count was detected. Similarly, the size distribution of MVs in the PFP stored at $-80^{\circ} \mathrm{C}$ for 1 or 4 weeks was comparable with that in the control samples, as shown in Fig. 4B, D, G and H.

$\mathrm{MVs}$ from EDTA-anticoagulated plasma stored at $4^{\circ} \mathrm{C}$ for 0 days (control), 3-4 days or 1 week exhibited no statistically significant differences in MV counts. Similarly, the size distribution showed no significant differences in the MVs that were 
A

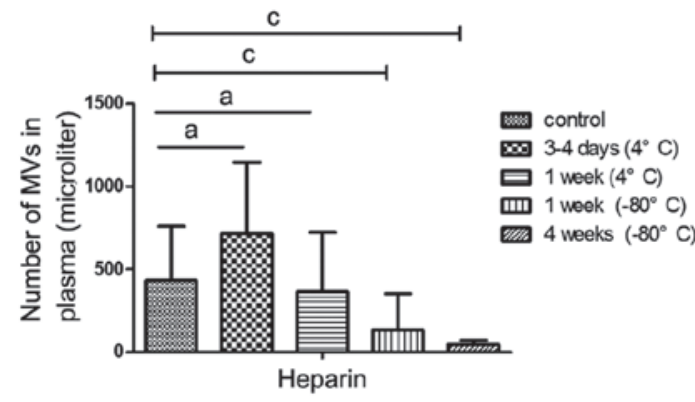

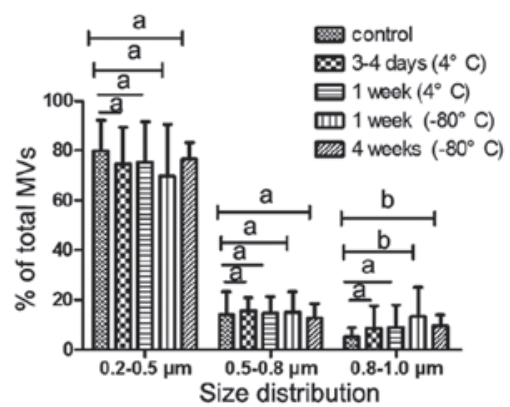
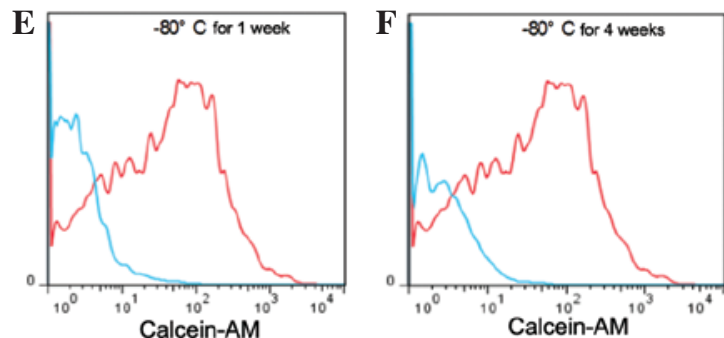

Figure 5. Effect of storage of MVs isolated from heparin-anticoagulated plasma at 4 or $-80^{\circ} \mathrm{C}$ for different periods. Impact on (A) $\mathrm{MV}$ counts and (B) size distribution, respectively. Representative histograms showing the impact of MV storage on MV counts vs. a control sample from a single individual are also presented (red lines, control; blue lines, experiment). Samples were stored at $4^{\circ} \mathrm{C}$ for (C) $3-4$ days or (D) 1 week, and at $-80^{\circ} \mathrm{C}$ for (E) 1 week or (F) 4 weeks. The control sample was collected and centrifuged immediately to isolate MVs, which were not stored. Data are expressed as the mean \pm standard error of the mean. ${ }^{a}$ No significance; ${ }^{b} \mathrm{P}<0.05 ;{ }^{\mathrm{c}} \mathrm{P}<0.01$. MV, microvesicle.

A

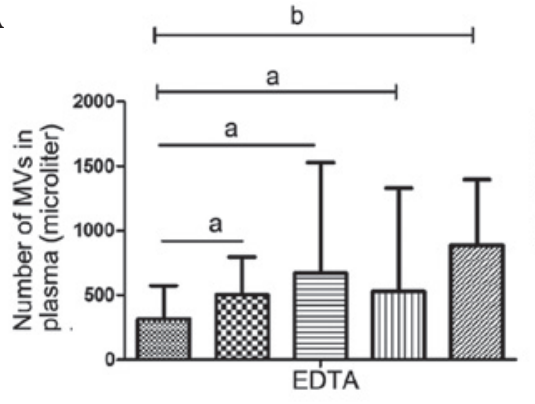

B

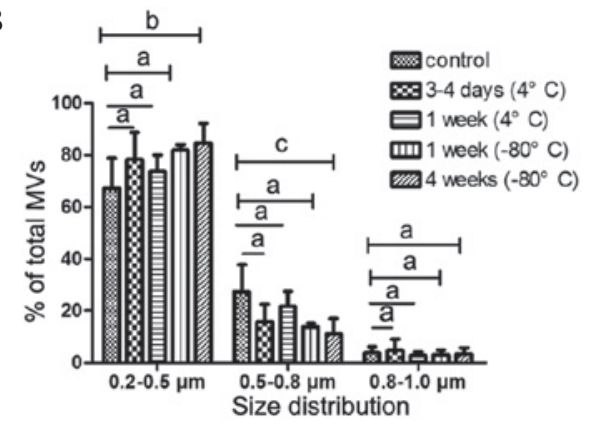

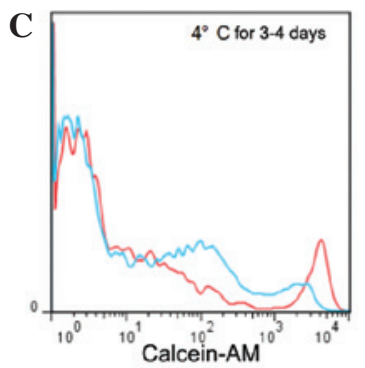
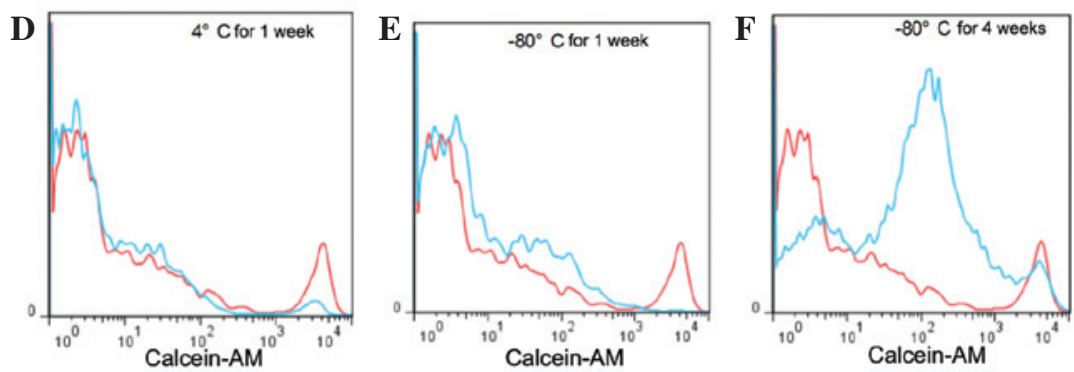

Figure 6. Impact of storage of MVs isolated from EDTA-anticoagulated plasma at 4 or $-80^{\circ} \mathrm{C}$ for different periods. Impact on (A) MV counts and (B) size distribution, respectively. Representative histograms showing the impact of MV storage on MV counts vs. a control sample from the same individual are also presented (red lines, control; blue lines, experiment). Samples were stored at $4^{\circ} \mathrm{C}$ for (C) $3-4$ days or (D) 1 week, and at $-80^{\circ} \mathrm{C}$ for (E) 1 week or (F) 4 weeks. The control sample was collected and centrifuged immediately to isolate MVs, which were not stored. Data are expressed as the mean \pm standard error of the mean. ${ }^{\mathrm{a}} \mathrm{No}$ significance; ${ }^{\mathrm{b}} \mathrm{P}<0.05 ;{ }^{\mathrm{c}} \mathrm{P}<0.01$. MV, microvesicle; EDTA, ethylenediaminetetraacetic acid.

stored at $4^{\circ} \mathrm{C}$ for up to 1 week (Fig. 6A-D), or those frozen at $-80^{\circ} \mathrm{C}$ for 1 week (Fig. 6A, B and E). However, MV counts and size were significantly influenced by freezing at $-80^{\circ} \mathrm{C}$ for 4 weeks (Fig. 6A, B and F). The number of MVs increased $(\mathrm{P}=0.041$ vs. control; Fig. $6 \mathrm{~A}$ and $\mathrm{F})$, whereas MV size gradually decreased. The percentage of MVs in the $0.5-0.8 \mu \mathrm{m}$ size distribution group significantly decreased $(\mathrm{P}=0.008$ vs. control) and the $0.2-0.5 \mu \mathrm{m}$ group increased ( $\mathrm{P}=0.014$ vs. control; Fig. 6B).
MV size distribution is affected by the anticoagulant used, but $M V$ count is not. Previous studies have reported differences in MV counts between heparin- and EDTA-anticoagulated plasma using Annexin V staining $(13,15)$; however, no data has been published to elucidate the impact of anticoagulant on MV counts following calcein-AM labeling. Therefore, the present study compared the counts and size distribution of MVs freshly isolated from plasma anticoagulated with heparin and EDTA. Notably, no statistically significant differences in the number 
A

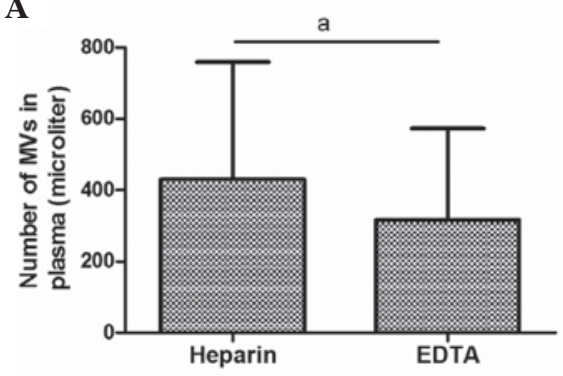

B

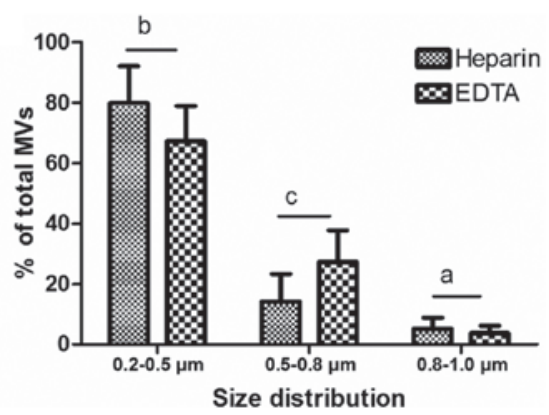

Figure 7. Impact of anticoagulant on (A) MV counts and (B) size distribution. ${ }^{\mathrm{a}}$ No significance; ${ }^{\mathrm{b}} \mathrm{P}<0.05$; ${ }^{\mathrm{C}} \mathrm{P}<0.01$. MV, microvesicle; EDTA, ethylenediaminetetraacetic acid.

of MVs were detected between the samples treated with these two anticoagulants. In the EDTA and heparin groups, MVs were predominantly distributed in the $0.2-0.5 \mu \mathrm{m}$ size group, to a reduced degree in the $0.5-0.8 \mu \mathrm{m}$ group and minimally in the $0.8-1.0 \mu \mathrm{m}$ group. However, the size of MVs in the EDTA-anticoagulated plasma was increased compared with that in the heparin-anticoagulated plasma: The percentage increased in the $0.5-0.8 \mu \mathrm{m}$ size distribution range $(\mathrm{P}=0.002)$ and decreased in the 0.2-0.5 $\mu \mathrm{m}$ group $(\mathrm{P}=0.032)$, compared with the size of MVs isolated from heparin-anticoagulated plasma (Fig. 7).

\section{Discussion}

In the present study, the effects of pre-analytical variables, including centrifugation speed and duration, and storage conditions on MV measurement using FCM with calcein-AM staining were evaluated. In addition, the impact of heparin and EDTA anticoagulants on MV counts and size distribution were compared. The data indicated that MV counts are independent of anticoagulant, centrifugation speed and duration, but are affected by storage conditions, while the size of MVs varied with plasma anticoagulant and storage conditions.

The profile of circulating MVs released by cells correlates with the development, progression and metastasis of a variety of cancers (24-26). According to the widely accepted principle that phosphatidylserine (PS) exposure on the cell surface is a common feature of MV release, Annexin V, a specific ligand to PS, is used for MV detection by FCM in the majority of experiments $(13,15,16)$. However, reports of the existence of Annexin V-negative MVs $(22,27,28)$ challenge the basis of this detection approach. Furthermore, PS exposure occurs in apoptosis and necrosis, which may lead to false-positive results using FCM. Thus, it is essential to identify a novel reagent for the detection of MVs. In the present study, calcein-AM was used to identify MVs, primarily due to the fact that it is colorless and nonfluorescent until hydrolyzed in cells by nonspecific esterases, and the leakage of the hydrolyzed compound out of cells is markedly slower compared with its parent compound, referred to the product introduction of calcein-AM. In addition, calcein-AM staining is independent of PS content and calcium concentration. The latter is a major drawback of Annexin V, leading to different results in MV counts between heparin- and EDTA-anticoagulated plasma. Therefore, calcein-AM may be suitable and relatively specific for intact MV labeling. To the best of our knowledge, the present study is the first to apply the calcein-AM staining method to label clinical circulating MVs. Further investigations are required to compare the differences in MV counts and size distribution between calcein-AM and Annexin V labeling in different conditions.

Previous studies have reported $(13,15)$ that MV counts are substantially reduced in blood when using EDTA compared with heparin; however, in the present study, no statistically significant differences in MV count were detected between EDTA and heparin using calcein-AM staining. A previous study suggested that calcium chelation by EDTA contributed to the decreased MV counts (15), while a different study attributed this difference to microvesiculation in vitro with heparin (16). The present data support the former explanation, indicating that the primary distinction between calcein-AM and Annexin V is the effect of calcium. In the present experiments that employed an MV staining reagent independent of calcium, no differences were observed in MV counts between samples treated with EDTA and heparin. EDTA has been recommended as the first line anticoagulant for hematological testing as it allows the optimal preservation of cellular components and morphology of blood cells (29). The results of the present study indicate that EDTA may be more suitable than heparin for MV detection by FCM, as EDTA preserves MV counts and size more effectively compared with heparin. Connor et al (30) suggested that, for blood samples that are not immediately processed, MV measurement should be performed using EDTA-anticoagulated samples (31), which is consistent with the results of the present study.

Isolation of MVs is a time-consuming process which increased the difficulty of large-scale sample preparation and detection. For this reason, it may be more practical and convenient if MVs could be obtained in a shorter time. The results of the present study comparing different centrifugation speeds and times may meet this requirement, thus assisting in batch processing of samples and promoting the development of MV detection. An excessive centrifugation speed may lead to fragment contamination of the MV pellet, so proper speed is critical for intact MV isolation. The present data indicated that centrifugation of PFP at 16,000 x g for 15 min was sufficient to achieve the requirements of MV isolation.

It may be optimal to proceed to isolation and detection of MVs immediately after plasma preparation. However, in certain cases, samples from different patients cannot be processed immediately and sample storage is necessary, which 
is usually as PFP or MVs $(13,15)$. Ayers et al (13) reported that $\mathrm{MV}$ counts were significantly reduced in MVs that were stored at $-80^{\circ} \mathrm{C}$ for an extended duration, which is consistent with the present results. This may be due to the adsorption of MVs to sample tubes, or MVs shrinking to a size that is below the detection limit of FCM for samples frozen at a low temperature for a long period. However, it appears that this explanation only applies to MVs from heparin-anticoagulated plasma, as the counts of MVs from EDTA-anticoagulated plasma increased in the MVs that were frozen at $-80^{\circ} \mathrm{C}$ for 4 weeks. The underlying mechanism remains unknown; however, it is plausible that this phenomenon may be associated with the calcium-chelating properties of the anticoagulant. PFP, rather than MVs, appears to be the preferable mode of sample storage, on the basis of the present results and the findings of previous experiments (15).

A number of studies have reported that MV size distribution modulates according to diverse stimuli in vitro $(20,21)$, indicating that MV size may be associated with disease condition or cause. Although previous studies have demonstrated the utility of electron microscopy, atomic force microscopy (AFM), dynamic light scattering (DLS) and microfluidics in measuring the size of circulating MVs $(2,32,33)$, these detection methods are relatively time-consuming and complicated. Compared with the aforementioned methods, FCM is user-friendly and is widely used in MV detection. However, little is known about MV size distribution as determined by FCM in samples from healthy tissues, or the effects of sample preparation methods. In the present study, calibration beads were used to define MV size and assess the impact of various preparation conditions on sample size distribution. MV size was predominantly $0.2-0.5 \mu \mathrm{m}$ in diameter, as observed using transmission electron microscopy (TEM), AFM or DLS $(32,33)$. However, MVs isolated from different anticoagulants exhibited diverse size distributions under different conditions. MVs from heparin-anticoagulated plasma frozen at low temperature were enlarged over time and presented with reduced counts, whereas the opposite was observed in the MVs stored in EDTA-anticoagulated plasma. For MVs from heparin-anticoagulated plasma, this may be a result of the aggregation of MVs into a reduced number of larger vesicles. For MVs from EDTA-anticoagulated plasma, larger MVs may have reduced to a size that is detectable using FCM. Notably, the average size of freshly isolated (i.e. never stored) MVs from EDTA-anticoagulated plasma is increased compared with that of MVs from heparin-anticoagulated plasma. Future investigations may be required in order to further elucidate these findings.

In conclusion, the present study evaluated the impact of pre-analytical variables including anticoagulant, centrifugation speed and time, and storage conditions on MV measurements using FCM with calcein-AM staining. Analysis of these factors is essential for the development of MVs as diagnostic and prognostic biomarkers for disease.

\section{Acknowledgements}

The authors would like to thank Yong Xu and Zhihui Liang from the Institute of Biochemistry (School of Basic Medicine, Huazhong University of Science and Technology, Wuhan, China) for their assistance in FCM detection and data analysis.
In addition, the authors thank the healthy volunteers for their cooperation in providing blood specimens for the present study. This study was supported by grants from the National Natural Science Foundation of China (nos. 81272624, 81300259 and 81170497).

\section{References}

1. Théry C, Ostrowski M and Segura E: Membrane vesicles as conveyors of immune responses. Nat Rev Immunol 9: 581-593, 2009.

2. Ashcroft BA, de Sonneville J, Yuana Y, Osanto S, Bertina R, Kuil ME and Oosterkamp TH: Determination of the size distribution of blood microparticles directly in plasma using atomic force microscopy and microfluidics. Biomed Microdevices 14: 641-649, 2012.

3. Dalton AJ: Microvesicles and vesicles of multivesicular bodies versus 'virus-like' particles. J Natl Cancer Inst 54: 1137-1148, 1975.

4. Keller S, Ridinger J, Rupp AK, Janssen JW and Altevogt P: Body fluid derived exosomes as a novel template for clinical diagnostics. J Transl Med 9: 86, 2011.

5. Hata T, Murakami K, Nakatani H, Yamamoto Y, Matsuda T and Aoki N: Isolation of bovine milk-derived microvesicles carrying mRNAs and microRNAs. Biochem Biophys Res Commun 396: 528-533, 2010.

6. Pisitkun T, Shen RF and Knepper MA: Identification and proteomic profiling of exosomes in human urine. Proc Natl Acad Sci USA 101: 13368-13373, 2004.

7. Runz S, Keller S, Rupp C, Stoeck A, Issa Y, Koensgen D, Mustea A, Sehouli J, Kristiansen G and Altevogt P: Malignant ascites-derived exosomes of ovarian carcinoma patients contain CD24 and EpCAM. Gynecol Oncol 107: 563-571, 2007.

8. Ghosh AK, Secreto CR, Knox TR, Ding W, Mukhopadhyay D and Kay NE: Circulating microvesicles in B-cell chronic lymphocytic leukemia can stimulate marrow stromal cells: Implications for disease progression. Blood 115: 1755-1764, 2010.

9. Bernal-Mizrachi L, Jy W, Jimenez JJ, Pastor J, Mauro LM, Horstman LL, de Marchena E and Ahn YS: High levels of circulating endothelial microparticles in patients with acute coronary syndromes. Am Heart J 145: 962-970, 2003.

10. Sellam J, Proulle V, Jüngel A, Ittah M, Miceli Richard C, Gottenberg JE, Toti F, Benessiano J, Gay S, Freyssinet JM and Mariette X: Increased levels of circulating microparticles in primary Sjögren's syndrome, systemic lupus erythematosus and rheumatoid arthritis and relation with disease activity. Arthritis Res Ther 11: R156, 2009.

11. Toth B, Liebhardt S, Steinig K, Ditsch N, Rank A, Bauerfeind I, Spannagl M, Friese K and Reininger AJ: Platelet-derived microparticles and coagulation activation in breast cancer patients. Thromb Haemost 100: 663-669, 2008

12. Galindo-Hernandez O, Villegas-Comonfort S, Candanedo F, González-Vázquez MC, Chavez-Ocana S, Jimenez-Villanueva X, Sierra-Martinez M and Salazar EP: Elevated concentration of microvesicles isolated from peripheral blood in breast cancer patients. Arch Med Res 44: 208-214, 2013.

13. Ayers L, Kohler M, Harrison P, Sargent I, Dragovic R, Schaap M, Nieuwland R, Brooks SA and Ferry B: Measurement of circulating cell-derived microparticles by flow cytometry: Sources of variability within the assay. Thromb Res 127: 370-377, 2011.

14. Ueba T, Haze T, Sugiyama M, Higuchi M, Asayama H, Karitani Y, Nishikawa T, Yamashita K, Nagami S, Nakayama T, et al: Level, distribution and correlates of platelet-derived microparticles in healthy individuals with special reference to the metabolic syndrome. Thromb Haemost 100: 280-285, 2008.

15. Jayachandran M, Miller VM, Heit JA and Owen WG: Methodology for isolation, identification and characterization of microvesicles in peripheral blood. J Immunol Methods 375: 207-214, 2012

16. Shah MD, Bergeron AL, Dong JF and López JA: Flow cytometric measurement of microparticles: Pitfalls and protocol modifications. Platelets 19: 365-372, 2008.

17. Breimo ES and Østerud B: Generation of tissue factor-rich microparticles in an ex vivo whole blood model. Blood Coagul Fibrinolysis 16: 399-405, 2005.

18. Piersma SR, Broxterman HJ, Kapci M, de Haas RR, Hoekman K, Verheul HM and Jiménez CR: Proteomics of the TRAP-induced platelet releasate. J Proteomics 72: 91-109, 2009. 
19. Jy W, Horstman LL, Jimenez JJ, Ahn YS, Biró E, Nieuwland R, Sturk A, Dignat-George F, Sabatier F, Camoin-Jau L, et al: Measuring circulating cell-derived microparticles. J Thromb Haemost 2: 1842-1851, 2004

20. Bernimoulin M, Waters EK, Foy M, Steele BM, Sullivan M, Falet H, Walsh MT, Barteneva N, Geng JG, Hartwig JH, et al: Differential stimulation of monocytic cells results in distinct populations of microparticles. J Thromb Haemost 7: 1019-1028, 2009.

21. Sun L, Wang HX, Zhu XJ, Wu PH, Chen WQ, Zou P, Li QB and Chen ZC: Serum deprivation elevates the levels of microvesicles with different size distributions and selectively enriched proteins in human myeloma cells in vitro. Acta Pharmacol Sin 35: 381-393, 2014.

22. Shet AS, Aras O, Gupta K, Hass MJ, Rausch DJ, Saba N, Koopmeiners L, Key NS and Hebbel RP: Sickle blood contains tissue factor-positive microparticles derived from endothelial cells and monocytes. Blood 102: 2678-2683, 2003.

23. Jayachandran M, Litwiller RD, Owen WG, Heit JA, Behrenbeck T, Mulvagh SL, Araoz PA, Budoff MJ, Harman SM and Miller VM Characterization of blood borne microparticles as markers of premature coronary calcification in newly menopausal women. Am J Physiol Heart Circ Physiol 295: H931-H938, 2008.

24. Yamada N, Tsujimura N, Kumazaki M, Shinohara H, Taniguchi K, Nakagawa Y, Naoe T and Akao Y: Colorectal cancer cell-derived microvesicles containing microRNA-1246 promote angiogenesis by activating Smad 1/5/8 signaling elicited by PML down-regulation in endothelial cells. Biochim Biophys Acta 1839: 1256-1272, 2014.

25. Zhang W, Zhao P, Xu XL, Cai L, Song ZS, Cao DY, Tao KS, Zhou WP, Chen ZN and Dou KF: Annexin A2 promotes the migration and invasion of human hepatocellular carcinoma cells in vitro by regulating the shedding of CD147-harboring microvesicles from tumor cells. PLoS One 8: e67268, 2013.
26. Al-Nedawi K, Meehan B, Micallef J, Lhotak V, May L, Guha A and Rak J: Intercellular transfer of the oncogenic receptor EGFRvIII by microvesicles derived from tumour cells. Nat Cell Biol 10: 619-624, 2008

27. Nielsen CT, Østergaard O, Johnsen C, Jacobsen S and Heegaard NH: Distinct features of circulating microparticles and their relationship to clinical manifestations in systemic lupus erythematosus. Arthritis Rheum 63: 3067-3077, 2011.

28. Joop K, Berckmans RJ, Nieuwland R, Berkhout J, Romijn FP, Hack CE and Sturk A: Microparticles from patients with multiple organ dysfunction syndrome and sepsis support coagulation through multiple mechanisms. Thromb Haemost 85: 810-820, 2001.

29. Banfi G, Salvagno GL and Lippi G: The role of ethylenediamine tetraacetic acid (EDTA) as in vitro anticoagulant for diagnostic purposes. Clin Chem Lab Med 45: 565-576, 2007.

30. Connor DE, Exner T, Ma DD and Joseph JE: Detection of the procoagulant activity of microparticle-associated phosphatidylserine using XACT. Blood Coagul Fibrinolysis 20: 558-564, 2009.

31. Yuana Y, Bertina RM and Osanto S: Pre-analytical and analytical issues in the analysis of blood microparticles. Thromb Haemost 105: 396-408, 2011.

32. György B, Módos K, Pállinger E, Pálóczi K, Pásztói M, Misják P, Deli MA, Sipos A, Szalai A, Voszka I, et al: Detection and isolation of cell-derived microparticles are compromised by protein complexes resulting from shared biophysical parameters. Blood 117: e39-e48, 2011.

33. Lawrie AS, Albanyan A, Cardigan RA, Mackie IJ and Harrison P Microparticle sizing by dynamic light scattering in fresh-frozen plasma. Vox Sang 96: 206-212, 2009. 